\title{
Surgical Repair of Secondary Subaortic Stenosis in Congenital Heart Disease Without Initial Subaortic Obstruction
}

\author{
Yuefeng Cao, MD, Xiangming Fan, MD \\ Department of Pediatric Cardiac Center, Beijing Anzhen Hospital, Capital Medical University, Beijing, China
}

\section{ABSTRACT}

Background: Secondary subaortic stenosis (SSS) is a rare heart disease of the left ventricular outflow tract (LVOT). It usually occurs after cardiovascular correction with or without initial left ventricular outflow tract obstruction (LVOTO). Because most patients with SSS are asymptomatic, many do not realize the need for reoperation until the obstruction worsens. Few studies suggest the characteristics and reasons of SSS without initial SAS. We conducted a retrospective study to describe the characteristics and surgical outcomes of these patients.

Methods: In this study, we examined a single-center retrospective cohort of SSS patients without initial SAS undergoing resection from 2010 to 2019. Patients are defined as secondary subaortic obstruction requiring surgery after cardiovascular correction. Demographics, perioperative findings, and clinical data were analyzed.

Results: Twenty-three patients had undergone secondary cardiac surgery for SSS without initial SAS during 10 years in our center. The median age at operation was 7.3 (4.0-13.5) years. In this study, the most commonly associated cardiac lesions were ventricular septal defect (VSD), atrioventricular septal defect (AVSD), patent ductus arteriosus (PDA), and coarctation arch hypoplasia (COA). The surgical techniques included membranous resection of five patients, fibromuscular resection of 17 patients, and reconstruction of the intraventricular baffle of one patient. The results of surgery in these patients are satisfied. The average LVOT gradient at the last follow up was $14.9(7.8-26.2) \mathrm{mmHg}$. There was no operative mortality. Two patients had postoperative complications. The median follow-up period was 2.9 (1.1-4.3) years with one late death. Two patients $(8.7 \%)$ had recurrence of stenosis.

Conclusions: Secondary subaortic stenosis is an uncommon heart disease. The reason is related to several causes, including missed diagnosis, unnoticed abnormalities of LVOT, and further changes of geometric morphology by intracardiac surgery. The results of surgery in these patients are satisfied. However, the recurrence of stenosis is still frequent.

Received September 14, 2021; accepted October 5, 2021.

Correspondence: Xiangming Fan, MD, Beijing Anzhen Hospital, No 2 Anzhen Road Chaoyang District, Beijing 100029, China (e-mail: fanxiangming@126.com).

\section{INTRODUCTION}

Subaortic stenosis (SAS) accounts for approximately $8-20 \%$ of left ventricular outflow tract (LVOT) obstruction in the young [Anderson 2017]. Secondary subaortic stenosis (SSS) usually occurs after heart defects with or without initial subaortic stenosis [Kalfa 2007]. It is known that the inadequate surgical resection of primary subaortic stenosis is the reason of SSS with initial SAS. But the SSS without initial SAS is not common in clinical practice. Due to the high rate of reoperation and mortality, SSS needs surgery as early as possible. So, SSS has become an important factor affecting the prognosis of patients after initial surgery.

Most studies [Anderson 2017; Sigfússon 1997; Brauner 1997] focus on analyzing recurrence factors of SAS, such as patient low age at the time of operation, the gradient of LVOT in preoperation, and so on. However, there are a lack of investigations about the reasons and outcomes of SSS, especially those patients without initial SAS. It is still controversial whether initial intracardiac surgery is related to the outcomes of surgical repair. Some study [Brauner 1997] shows that the morphologic abnormalities and congenital heart defects are risk factors for the development of SSS. They affect septal shear stress in the direction and magnitudes that have been linked to cellular growth factors. We reviewed our surgical results in the recent era to investigate the clinical characteristics and surgical outcomes of SSS without initial SAS and to provide treatment options for these patients.

\section{PATIENTS AND METHODS}

Study population: The retrospective study of surgical records was performed in our institution for SSS between April 2010 and November 2019 (22983 patients with cardiac surgery). The inclusion criterion is the appearance, after congenital cardiovascular surgery, of a secondary subaortic obstruction requiring surgery. The main indications for surgery were LVOT obstruction and LVOT gradient greater than $40 \mathrm{mmHg}$. When the LVOT gradient was lower than $40 \mathrm{mmHg}$, there were symptoms (fatigue, chest pain, and syncope), and surgery was necessary. The criterion for exclusion was a subaortic stenosis at the time of the first operation. Patients with initial LVOT obstruction, severe aortic stenosis, single ventricle anatomy, and hypertrophic cardiomyopathy also were excluded. Thus, 23 patients were studied. 
Data collection: Data were extracted from the electronic medical record system. The baseline data collected for these 23 patients included demographic, anatomical characteristics, and SSS subtype. Surgical parameters included surgical technique, extracorporeal circulation time (CPB time), aortic cross-clamp time (AXC-time), mechanical ventilation time, and ICU stay. Echocardiographic parameters included LVOT gradient (time before and after operation). Clinical and echocardiographic follow-up data were obtained in all patients.

Ethical approval: Given the retrospective design of the study, which is based on data collected for routine clinical care, administrative, and audit purposes, individual informed consent was not required. All procedures performed in this study were in accordance with the ethical standards of the institutional and/or national research committee and with the 1964 Helsinki Declaration and its later amendments or comparable ethical standards. This study has approval by the institutional research committee at Beijing Anzhen Hospital, which permitted the collection of data for audit and research purposes.

Statistical analysis: All data are analyzed using SPSS 24.0 software. Quantitative data are given as mean standard deviation or median (range). The symmetrically distributed data were expressed as mean \pm standard deviation $(\mathrm{SD})$, and the asymmetrically distributed data were expressed as interquartile range between the 25 th and 75 th percentile. Qualitative values are expressed as percentages. The t-test is used to compare preoperative and postoperative measurements. All statistical tests with a value of $P<0.05$ are considered significant.

\section{RESULTS}

Baseline characteristics: In our center, 65 patients had an SAS requiring surgery. Twenty-three patients underwent second cardiac surgery for SSS without initial SAS. (Table 1) There were $13(56.5 \%)$ males. The median age at operation was $7.6(6-13)$ years. The intervals time of two operations was $7(5-10)$ years. There were five patients with membranous, one patient with accessory mitral valve attachments, and 17 patients with fibromuscular. The median CPB time was $98 \pm 37.2 \mathrm{~min}$, and the median aortic cross-clamping time was $46(32-58) \mathrm{min}$. The mechanical ventilation time was 11 (6-16.8) hours, and the ICU time was 2 (1-2) days.

There were 20 patients with intracardiac surgery in initial operation. The initial heart defects are described in Figure 1. (Figure 1) The most commonly associated cardiac lesions were VSD $(47.8 \%, N=11)$, AVSD $(26.1 \%, N=6)$, PDA $(21.7 \%, N=5)$, COA $(13 \%, N=3)$, LSVC $(8.7 \%, N=2)$, and DORV $(8.7 \%, N=2)$. Meanwhile, there were $11(47.8 \%)$ patients with aortic mild regurgitation and four (17.4\%) patients with aortic moderate regurgitation.

For surgical technique, five patients underwent membranous resection, including two patients without initial intracardiac surgery and three patients with initial intracardiac surgery. Fibromuscular resection was used in 17 patients, including 16 patients without initial intracardiac surgery and one patient with initial intracardiac surgery. There was one patient (3.8\%) without initial intracardiac surgery who received the reconstruction of intraventricular baffle. This patient underwent a prosthetic valve implantation and used the dacron patch to enlarge the left ventricular outflow tract. The gradient of LVOT before and after the procedure was 63 and $23 \mathrm{mmHg}$, respectively.

The average pressure gradient of these patients decreased from $92.6 \pm 27.6 \mathrm{mmHg}$ to $15.3(9.3-26) \mathrm{mmHg}$. (Figure 2) There were no in-hospital deaths. One major postoperative complication included complete heart block requiring a pacemaker in two patients.

The average follow-up time was 2.9 (1.1-4.3) years. In all patients, the average LVOT gradient at the last follow up was $14.9(7.8-26.2) \mathrm{mmHg}$. The probability of recurrence was $8.7 \%$ after SSS repair. Two patients had recurrence of stenosis. One patient was a 6-year-old boy with VSD. His LVOT gradient after the operation and follow-up time was 35 and 46 $\mathrm{mmHg}$, respectively. Another patient was a 10 -year-old boy with IAA and VSD. His LVOT gradient after the operation and follow-up time was 49 and $81 \mathrm{mmHg}$, respectively. The mortality rate was $4.3 \%$ in follow-up time. A 7.6-year-old boy with VSD and LSVC died suddenly after 5.6 years reoperation. His LVOT gradient before and after the operation weas 78 and $35 \mathrm{mmHg}$, respectively.

\section{DISCUSSION}

This retrospective study contains 23 patients who had undergone SSS repair from a single institution. It investigates the causes and surgical outcomes of SSS without initial SAS. Some studies [Mukadam 2018; Valeske 2011; Ruzmetov 2006] showed that pathophysiological substrate of heart malformation offered valuable information about the subaortic stenosis long-term prognosis after cardiac surgery. In this study, we found that SSS without initial SAS was mainly related to the initial abnormal geometric and the further changes of geometric morphology by intracardiac surgery. The most commonly associated cardiac lesions were VSD, AVSD, PDA, and COA. Although patients had satisfactory surgical outcomes in this study, two patients had recurrence of subaortic stenosis.

The abnormal geometry of LVOT in initial surgery is the main risk of SSS. Some patients may be missed diagnosis or unnoticed abnormalities of LVOT in initial surgery. Eleven patients ( $42.3 \%$ ) had VSD in our study. The shape and size of VSD may interfere with the detection and resection of lesion, especially if echocardiograph does not accurately appreciate it. Supporting this finding is the result of Brauner et al. [Brauner 1997], who agreed that a vast majority of restrictive VSDs may be difficult both clinically and echocardiographically to accurately appreciate a subaortic obstructive lesion. Furthermore, the average interval time of two operations was 6 years in these patients. These patients may have had the abnormal geometry of LVOT in initial surgery, but this did not attract the attention of the surgeon. Cape [Brauner 1997] suggested that the presence of a VSD caused marked augmentation of the elevated shear stress produced by aortoseptal angle. It explained the high association of VSD and SSS. 
Table 1. Characteristics of patients with SSS

\begin{tabular}{|c|c|c|c|c|c|c|}
\hline 1 & COA, PDA & AAA & PLSVC & membranous resection & 45 & 12 \\
\hline 3 & $\mathrm{COA}$ & AAA & - & membranous resection & 119 & 24 \\
\hline 4 & DORV & IV repair & - & fibromuscular resection & 169 & 20 \\
\hline 6 & IAA, VSD, PDA & VSD closure, AAA & - & fibromuscular resection & 80 & 49 \\
\hline 7 & PAVSD & Repair of PAVSD & - & membranous resection & 108 & 22 \\
\hline 8 & PAVSD & Repair of PAVSD & - & membranous resection & 61 & 5 \\
\hline 9 & PAVSD & Repair of PAVSD & - & $\begin{array}{l}\text { accessory mitral valve } \\
\text { tissue resection }\end{array}$ & 98 & 6 \\
\hline 12 & CAVSD & Repair of CAVSD & - & fibromuscular resection & 81 & 20 \\
\hline 13 & TOF & Repair of TOF & - & fibromuscular resection & 81 & 5 \\
\hline 14 & VSD, ASD & VSD, ASD closure & - & fibromuscular resection & 61 & 9 \\
\hline 15 & VSD & VSD closure & MVR & fibromuscular resection & 77 & 36 \\
\hline 16 & VSD, PDA & VSD, PDA closure & - & fibromuscular resection & 140 & 12 \\
\hline 17 & VSD, PDA & VSD, PDA closure & - & fibromuscular resection & 103 & 15 \\
\hline 18 & VSD, PDA & VSD, PDA closure & - & fibromuscular resection & 84 & 7 \\
\hline 19 & VSD & VSD closure, mitral valve repair & supravalvular mitral ring & fibromuscular resection & 60 & 29 \\
\hline 20 & VSD & VSD closure & PLSVC & fibromuscular resection & 75 & 38 \\
\hline
\end{tabular}

AAA, aortic arch angioplasty; ASD, atrial septal defect; CAVSD, complete atrioventricular septal defect; COA, coarctation of the aorta; DORV, double outlet right ventricle; IV, intraventricular; MVR, mitral valve regurgitation; PAPVC, partial anomalous pulmonary venous connection; PAVSD, partial atrioventricular septal defect; PDA, patent ductus arteriosus; RVOTS, right ventricular outflow tract stenosis; TOF, Tetralogy of Fallot; VSD, ventricular septal defect

Additionally, two patients underwent previous operations for COA. Some studies [Hirata 2009; Alsoufi 2016] suggested morphologic abnormalities of COA in the LVOT might result in altered septal shear stress, which can lead to cell proliferation and recurrent obstruction in the LVOT. Although five patients had PDA, but it was still uncertain if the defect was associated with subaortic stenosis. And so far, there is no study about this area.

SSS is related to the further changes of geometric morphology by intracardiac surgery, such as AVSD, IAA and DORV. The occurrence of SSS may be related to the changes of LVOT geometric morphology after cardiac defects repair. Some study has shown that SSS is associated with defects, such as VSD or conotruncal anomalies in $60 \%$ of cases and may develop after patch closure of a perimembranous or malaligned VSD [Kalfa 2007]. In this study, 10 patients may have the further changes of geometric morphology by surgery
(6 AVSD, 2 DORV, 1 TOF, and 1 IAA). These patients had no subaortic stenosis during the early postoperative period. The average interval time of two operations was 11.8 years. The morphology abnormalities of AVSD are the main risk of SSS. Its main feature includes a "goose neck" deformity, which creates a narrow and elongated LVOT with an abnormal outlet angle [Buratto 2016; Overman 2014]. In this study, six cases $(26.1 \%)$ after AVSD repair occurred SSS resection, including two patients with membrane resection and four patients with fibromyotomy resection. In addition, one patient with SSS after AVSD repair, which was not included this study, had successful surgery results, using the method of releasing the secondary chordae of the mitral anterior leaflet. Due to posterior deviation of the conal septum, the risk of SSS increased in patients with IAA. Several surgical strategies have been proposed to decrease obstruction risk at time of initial IAA repair. Those surgical strategies included 


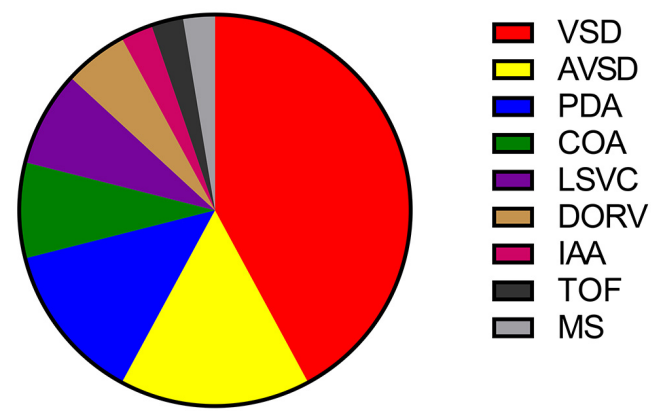

Initial heart defect

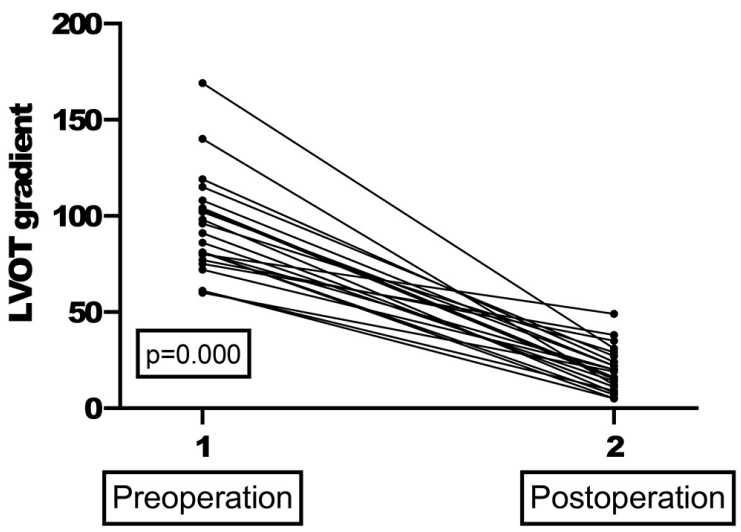

Figure 2. LVOT pressure gradient in preoperation and postoperation concomitant resection of the conal septum, Ross Konno, or Damus-Kaye-Stansel connection to completely bypass the narrow left ventricular outflow tract [Alsoufi 2016].

SSS also is an iatrogenic disease after biventricular repair of DORV. SSS after biventricular repair of DORV may develop in 3.5-5.5\% of cases [Zhang 2020]. The changes in geometric morphology, such as the decrease in the effective VSD size, turbulent flow, and the sinuous shape of the tunnelization, caused subaortic septal or conal muscle hypertrophy or fibrous tissue deposits [Zhang 2020; Kim 2010]. The turning radius of the intraventricular tunnel is the main factor, affecting the pressure difference. A small turning radius of the intraventricular tunnel will lead to a large pressure difference in the intraventricular tunnel [Yang 2020]. The surgical techniques used for SSS repair include the extended septoplasty and the fibromuscular resection. One patient with DORV underwent the extended septoplasty after 11 years of biventricular repair in this study. The extended septoplasty was a safe and effective method for the treatment of subaortic stenosis, especially in cases with a long-tunnel shaped LVOTO [Kim 2010]. The postoperative LVOT gradient of these patients was significantly lower than before surgery in this study. The fibromuscular ridge resection $(73.9 \%, N=17)$ was the most common technique. Brauner et al. [Brauner 1997] suggested that the routine addition of a generous myectomy to fibrous subaortic resection for relief of tunnel LVOT obstruction may reduce the incidence of recurrent obstruction. It can minimize the incidence of restenosis and ensured that the LVTO is sufficiently unobstructed. Meanwhile, when abnormal fibrous tissue extends or covered the anterior mitral valve leaflet, complete removal of abnormal fibrous tissue from the LVOT is important to prevent the recurrence of subaortic stenosis. It is important to avoid injury to the conduction system. In our study, two patients of our study had complete heart block after fibromuscular ridge resection and required pacemakers (one DORV and one TOF) implantation.

Only five patients $(21.7 \%$ ) had symptoms in this study, including fatigue, chest pain, or syncope. Most patients were diagnosed SSS during follow-up time. This showed that close follow up is particularly important for patients after initial heart surgery. Furthermore, for those patients undergoing initial operations of heart defects, the abnormalities of LVOT geometric morphology should have received more attention. Although the surgical results are satisfactory, there still are stenosis recurrences. Recurrence of subaortic stenosis after surgery is common in children. Mukadam et al. [Mukadam 2018] showed that recurrence of subaortic stenosis within several years after initial surgical treatment was frequent in patients with complex lesion. Two patients (8.7\%) had recurrence of SSS in our study. The reason of restenosis may be the unsatisfied resection. The hemodynamic changes caused by the remaining diaphragm can cause restenosis. For these patients, complete removal of abnormal fibrous tissue and regular follow up is particularly important. Due to insufficient clinical evidence, the cause of death is not yet clear.

Limitations: This investigation was a retrospective review from a single institution without standardized echocardiographic review and detailed imaging data. The decisions for surgical intervention and reintervention were determined by individual practitioners and with no standardized criteria. These limited the analysis of risk factors for SSS without initial SAS. Further research is needed to validate these echocardiographic predictors in a larger prospective study. Also, the median follow-up time is relatively short, and stronger inferences could be made with longer follow up.

\section{CONCLUSION}

SSS without initial SAS is not common in LVOT. It is mainly related to several causes, includes missed diagnosis, unnoticed abnormalities of LVOT, and the further changes of geometric morphology by intracardiac surgery. Patients had satisfied surgical outcomes in this study. However, the recurrence of subaortic stenosis after surgery is uncommon. More elaborate and longer follow up is needed in these patients.

\section{REFERENCES}

Alsoufi B, Schlosser B, McCracken C, Sachdeva R, Kogon B, Border $\mathrm{W}$, et al. 2016. Selective management strategy of interrupted aortic arch 
mitigates left ventricular outflow tract obstruction risk. J Thorac Cardiovasc Surg. Feb;151(2):412-20.

Anderson BR, Tingo JE, Glickstein JS, Chai PJ, Bacha EA, Torres AJ. 2017. When Is It Better to Wait? Surgical Timing and Recurrence Risk for Children Undergoing Repair of Subaortic Stenosis. Pediatr Cardiol. Aug;38(6):1106-1114.

Brauner R, Laks H, Drinkwater DC, et al. 1997. Benefits of Early Surgical Repair in Fixed Subaortic Stenosis. J Am Coll Cardiol. Dec;307(7).

Brauner R, Laks H, Drinkwater DC, Shvarts O, Eghbali K, Galindo A. 1997. Potential Role of Mechanical Stress in the Etiology of Pediatric Heart Disease: Septal Shear Stress in Subaortic Stenosis. J Am Coll Cardiol. Dec;30(7):1835-42.

Buratto E, Ye XT, Bullock A, Kelly A, d'Udekem Y, Brizard CP, et al. 2016. Long-term outcomes of reoperations following repair of partial atrioventricular septal defect. Eur J Cardiothorac Surg. Aug; 50(2):293-7.

Hirata Y, Chen JM, Quaegebeur JM, Mosca RS. 2009. The role of enucleation with or without septal myectomy for discrete subaortic stenosis. J Thorac Cardiovasc Surg. May;137(5):1168-72.

Kalfa D, Ghez O, Kreitmann B, Metras D. 2007. Secondary subaortic stenosis in heart defects without any initial subaortic obstruction: a multifactorial postoperative event. Eur J Cardiothorac Surg. Oct;32(4):582-7.

Kim CY, Kim WH, Kwak JG, Jang WS, Lee CH, Kim DJ, Lim C, et al. 2010. Surgical Management of Left Ventricular Outflow Tract Obstruction after Biventricular Repair of Double Outlet Right Ventricle. J Korean Med Sci. Mar; 25(3):374-9.
Mukadam S, Gordon BM, Olson JT, Newcombe JB, Hasaniya NW, Razzouk AJ, et al. 2018. Subaortic Stenosis Resection in Children: Emphasis on Recurrence and the Fate of the Aortic Valve. World J Pediatr Congenit Heart Surg. Sep;9(5):522-528.

Overman DM. 2014. Reoperation for Left Ventricular Outflow Tract Obstruction After Repair of Atrioventricular Septal. Semin Thorac Cardiovasc Surg Pediatr Card Surg Annu. 171(1).

Ruzmetov M, Vijay P, Rodefeld MD, Turrentine MW, Brown JW. 2006. Long-term results of surgical repair in patients with congenital subaortic stenosis. Interact Cardiovasc Thorac Surg. Jun;5(3):227-33.

Sigfússon G, Tacy TA, Vanauker MD, Cape EG. 1997. Abnormalities of the Left Ventricular Outflow Tract Associated with Discrete Subaortic Stenosis in Children: An Echocardiographic Study. J Am Coll Cardiol. Jul;30(1):255-9.

Valeske K, Huber C, Mueller M, Böning A, Hijjeh N, Schranz D, et al. 2011. The Dilemma of Subaortic Stenosis - A Single Center Experience of 15 Years with a Review of the Literature. Thorac Cardiovasc Surg. Aug;59(5):293-7.

Yang Y, Wang J, Qiao A, Fan X. 2020. Numerical Simulation of the Influence of Geometric Configurations on Pressure Difference in the Intraventricular Tunnel. Front Physiol. Feb 21;11:133.

Zhang X, Wang W, Yan J, Wang Q. 2020. Surgical treatment results of secondary tunnel-like subaortic stenosis after congenital heart disease operations: A 7-year, single-center experience in 25 patients. J Card Surg. Feb; 35(2):335-340. 